\title{
Rabdomiosarcoma embrionario del cordón espermático asociado a adenocarcinoma prostatico. Presentación de un caso y revisión de la literatura
}

\author{
Capdevila Querol S, Jurado Troyano I*, Lamas moure S**, Aguilar Ruiz A, Alcoberro Turu A, \\ Bernal dzekonski G.
}

Unidad de Urologia. *Servicio de Anatomia Patológica. **Servicio de Cirugia General. Consorcio Sanitario de Terrassa.

Actas Urol Esp. 2008;32(9):945-947

\section{RESUMEN}

RABDOMIOSARCOMA EMBRIONARIO DEL CORDÓN ESPERMÁTICO ASOCIADO A ADENOCARCINOMA PROSTATICO. PRESENTACIÓN DE UN CASO Y REVISIÓN DE LA LITERATURA

Presentamos el caso de una tumoración derivada de estructuras del cordón espermático. La histología (rabdomiosarcoma), la edad de presentación (adulta) y la asociación con un cáncer de próstata motivó esta nota clínica. La especificidad del estudio anatomopatológico e inmunohistoquímico y el planteamiento del tratamiento regional son los dos aspectos claves del caso.

Palabras clave: Cordón espermático. Sarcoma.

\section{ABSTRACT \\ EMBRYONAL RHABDOMYOSARCOMA OF SPERMATIC CORD AND PROSTATIC ADENOCARCINOMA. CASE REPORT AND REVIEW OF THE LITERATURE}

We present a tumor developed from spermatic cord structures. The histology (rhabdomyosarcoma), the age of the patient and its association with a prostatic adenocarcinoma has developed this case report.

Histopathological singularity and regional treatment choice are the two key points of this case. Keywords: Spermatic cord. Sarcoma.

$\mathrm{E}$ Rabdomiosarcoma embrionario del cordón espermático es una tumoración infrecuente, especialmente en adultos. La asociación con un adenocarcinoma prostático en nuestro caso, lo hace aún más especial.

La excepcionalidad de dicha tumoración, su potencial agresivo y la tendencia a la recurrencia locorregional; han hecho que en los últimos años se intente establecer un protocolo de actuación integrado (quirúrgico y quimioradioterápico) con el objetivo de consensuar una misma línea de actuación.

\section{CASO CLÍNICO}

Paciente de 67 años de edad, sin alergias medicamentosas, con antecedentes de historia de taba- quismo, enolismo moderado y herniorrafia inguinal bilateral; que consultó en nuestra unidad por cuadro de tumoración indolora inguinoescrotal derecha de tres meses de evolución.

A la exploración física se apreció masa pétrea que ocupaba canal inguinal derecho hasta escroto, sin poder valorar teste derecho. Área inguinal izquierda libre, no hernias. Pene y teste izquierdo sin hallazgos, ausencia de adenopatías inguinales palpables. Al tacto rectal se apreció próstata mediana, con incremento de consistencia bilateral.

Se procedió a estudio mediante:

- Analítica: PSA 27 ng/mL. Marcadores tumorales testiculares, bioquímica y hemograma, dentro de la normalidad. 
- Tc Abdomen: (Fig. 1) : Destaca la presencia de masa de 15 por $8 \mathrm{~cm}$. que se inicia en orificio inguinal externo derecho y finaliza en bolsa escrotal derecha desplazando caudalmente al testículo derecho. Presencia de adenopatías (infracentiméricas) ilíacas comunes bilaterales e ilíacas externas derechas.

- Tc Torácico: Sin hallazgos patológico.

- GGO: Negativo para metástasis.

- Biopsia de Próstata: Adenocarcinoma de próstata bilateral gleason 7 .

Se procedió a exéresis de tumoración, orquiectomía derecha, funiculectomia radical derecha y linfadenectomia iliaca derecha; con cierre del defecto de la pared abdominal mediante malla de Prolene.

El estudio histopatológico diagnostica la tumoración de Rabdomiosarcoma Embrionario de células fusiformes (Figs. 2, 3 y 4), de $18 \mathrm{~cm}$ de diámetro. Márgenes de resección libres. Linfadenectomia negativa.

Se completó tratamiento con Radioterapia Locorregional (RL) y Bloqueo Androgénico Completo; negándose el enfermo a realizar pauta de quimioterapia propuesta (IVE: Ifosfamida-Vincristina-Etoposido).

Evolución posterior: progresión ganglionar finalmente aceptó quimioterapia. Revalorando tratamiento quimioterápico en paciente de 67 años con rabdomiosarcoma embrionario en progresión ganglionar pélvica se decide tratamiento con quimioterapia alternante según siguiente régimen:

$1^{\circ}$ : Ifosfamida-Epirrubicina $(2 \mathrm{~g} / \mathrm{m} 2 \times 3 \mathrm{~d}-45$ $\mathrm{mg} / \mathrm{m} 2 \times 2$ ) cada 21 días por 2 ciclos. Alternando con $2^{\circ}$ : Ifosfamida-Vincristina-Etoposido $(2 \mathrm{~g} / \mathrm{m} 2 \mathrm{x}$ $3 \mathrm{~d}-2 \mathrm{mg} \mathrm{d} 1-100 \mathrm{mg} / \mathrm{m} 2 \times 3$ ).

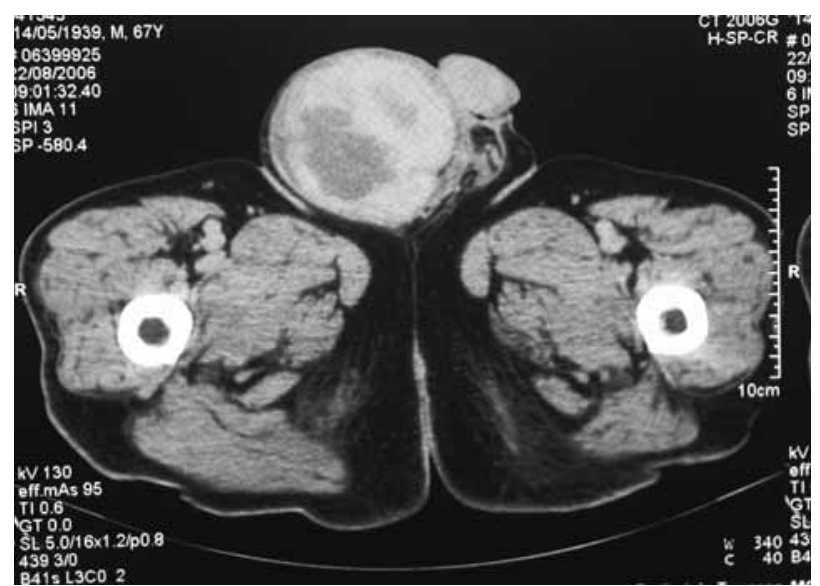

FIGURA 1. Tc Abdomen. Masa sólida con componente hemorrágico que se incia en orificio inguinal externo alcanzando hemiescroto derecho.

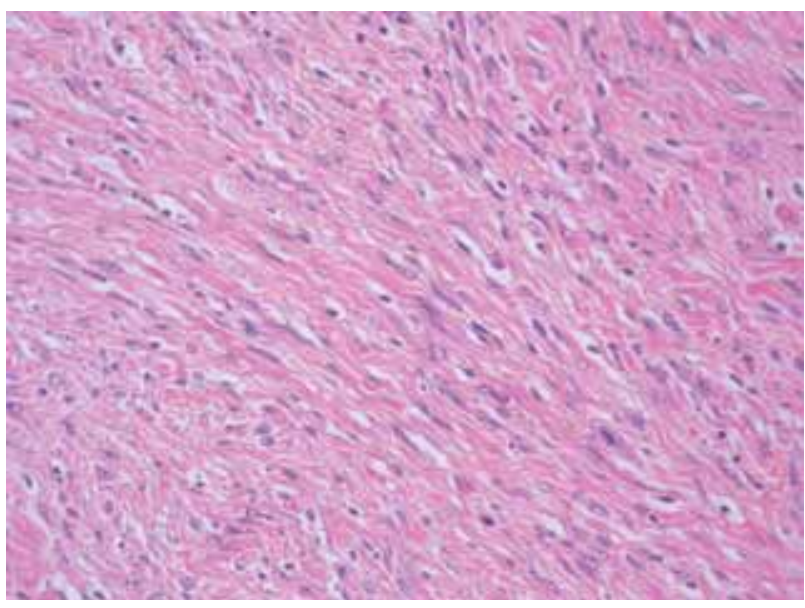

FIGURA 2. Histología. H y E. Area tumoral predominante de caracteristicas fusocelular.

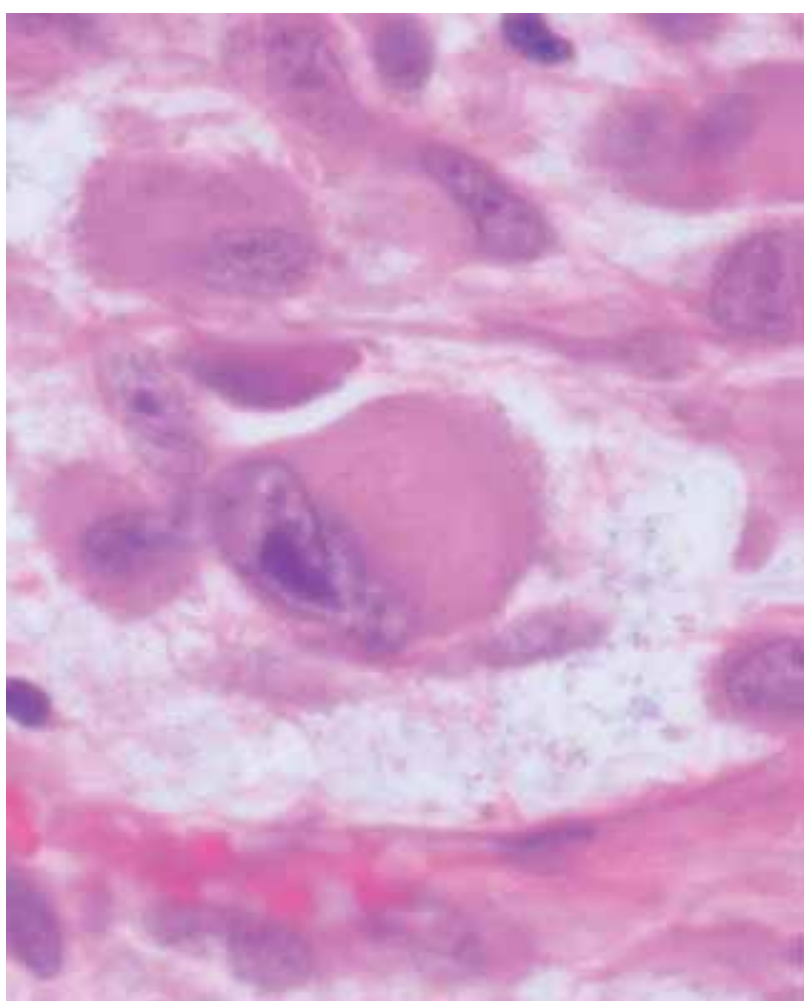

FIGURA 3. Histología. H y E. detalle de Rabdomioblasto.

\section{DISCUSION}

Los sarcomas representan el $1 \%$ de todas las neoplasias y en torno a un 2-3 \% afectan al aparato genitourinario $^{1-3}$. La afectación paratesticular es la más frecuente y de mejor pronóstico que en otras localizaciones (vejiga, riñón o próstata). La mayoría de tumoraciones paratesticulares dependen de estructuras del cordón espermático, las dos malignas más frecuentes son de origen mesodérmico y son el sarcoma y el rabdomiosarcoma. 


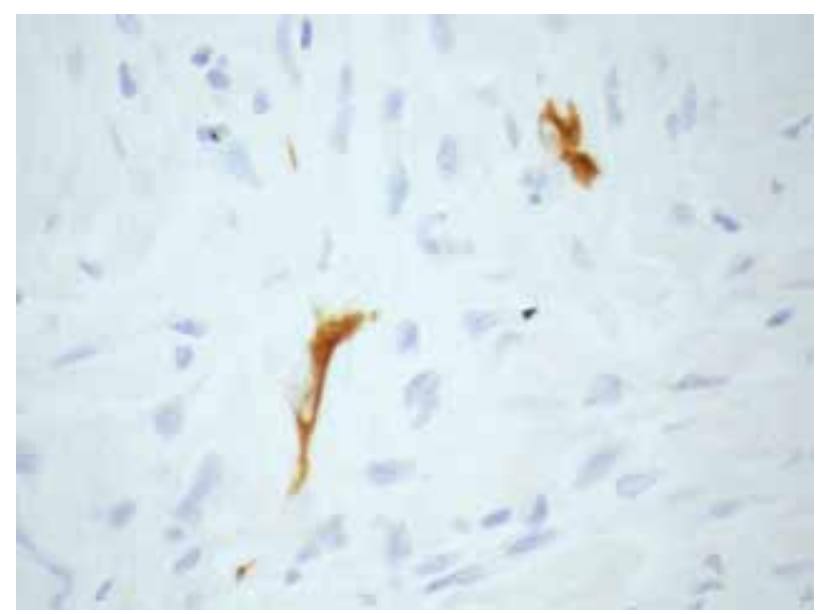

FIGURA 4. Histología. Inmunohistoquimica. Tinción de Desmina positivo en células tumorales fusiformes.

El Rabdomiosarcoma del cordón espermático fue descrito por primera vez por Rokitansky en $1849^{4}$ ) y tiene su mayor incidencia (60\%) en torno a las dos primeras décadas de la vida; suele manifestarse como una tumoración indolora inguinoescrotal que desplaza caudalmente el testículo. La supervivencia actual es aproximadamente de un 75\% a los 5 años, con una elevada tendencia a la recurrencia locorregional (50\%). Se describen diversos Subtipos Histológicos: Embrionario (el de mejor pronóstico), Pleomórfico (el más frecuente en adultos), Alveolar y Mixto.

El patrón embrionario fusiforme $(4,4 \%)^{5}$ y la edad de presentación típica confiere mejor pronóstico. La afectación en adultos, el tamaño tumoral y la afectación ganglionar confieren peor pronóstico ${ }^{3}$.

La clasificación (Tabla 1) establecida por el Intergroup rhabdomyosarcoma study (IRS), permite estadificar la afectación tumoral y establecer protocolos de actuación integrados.

El tratamiento local consiste en la exéresis de la tumoración, la orquiectomía y la funiculectomia amplia.

Tabla 1. Clasificación por estadios según el intergrupo rhabdomyosarcoma study (IRS)

IRS I: Tumoración localizada y resecada por completo.

IRS II: Resección completa pero evidencia de diseminación regional.

IRS III: Resección incompleta con enfermedad residual macroscópica.

IRS IV: Metástasis a distancia en el momento del diagnóstico.
El tratamiento regional ${ }^{6}$ (Linfadenectomia retroperitoneal [LRP] vs. Radioterapia [RDT]), es actualmente el que suscita mas controversia. La LRP permite estadificar correctamente la enfermedad y su morbilidad se ha reducido en los últimos años (gracias a la estandarización en el cáncer de testículo). Por otro lado la RDT en pacientes en edad pediátrica suscita el temor de la aparición de segundas neoplasias en un futuro.

El tratamiento sistémico ${ }^{7,8}$ : El Rabdomiosarcoma paratesticular se considera una enfermedad sistémica y como tal necesita de un tratamiento sistémico quimioterápico con regímenes que incluyan la Ciclofosfamida, Doxorrubicina y Vincristina. En pacientes de edad adulta se establecen protocolos en que incluyen Etoposido e Ifosfamida.

Actualmente en $3^{\text {er }}$ ciclo de QMT, con buena tolerancia. Última visita el 21 de marzo. Seguimiento en 9 meses.

\section{REFERENCIAS}

1. Mondaini N, Palli D, Saieva C, Nesi G, Franchi A, Ponchietti R, Tripodi S. Clinical characteristics and overall survival in genitourinary sarcomas treatec with curative intent: A Multicenter Study. Eur Urol. 2005;47(4):468-473.

2. Rodríguez García N, Llanes González L, Pascual Mateo C, Berenguer Sánchez A. Rabdomiosarcoma de cordón espermático en el adulto Arch Esp Urol. 2005;58(9):956-959.

3. Moroni M, Nesi G, Travaglini F, Rizzo M, Amorosi A, Dominici A. Rhabomyosarcoma of the Spermatic Cord Urol Int. 2003;71(1): 114-117.

4. Hoekstra H, Wobbes T, Brouwers T et al. Embryonal Rhabdomyosarcoma of spermatic cord. Urology. 1980;16(4):360-363.

5. Kizer WS, Dykes TE, Brent EL, Chatham JR, Schwartz BF. Spindle Cell Rhabdomyosarcoma in an adult. J Urol. 2001;166 (2):606-607.

6. Hermans BP, Foster RS, Bihrle R, Little S, Sandler A, Einhorn $\mathrm{LH}$ et al. Is retroperitoneal lymph node dissection necessary for adult paratesticular rhabomyosarcoma?. J Urol. 1998;160(6 Pt 1):2074-2077.

7. Wolden SL, Anderson JR, Crist WM, Breneman JC, Wharam MD $\mathrm{Jr}$, Wiener ES et al. Indications for radiotherapy and chemotherapy after complete resection in Rhabdomyosacoma: A report from the intergruop Rhabdomyosarcoma Studies I to III. J Clin Oncol. 1999(11):3468-3475.

8. Crist WM, Anderson JR, Meza JL, Fryer C, Raney RB, Ruymann FB et al. An Intergropu Rhabdomyosarcoma Study- IV: Results for Patients with nonmetastatic Disease. J Clin Oncol. 2001;19 (12):3091-3102.

Correspondencia autor: Dr. S. Capdevila Querol

Unidad de Urología. Consorcio Sanitario de Terrasa

Carretera de Torrebonica, s/n - 08227 Terrasa (Barcelona)

Tel.: 937310007

E-mail autor: scq33020@hotmail.com

Información artículo: Nota clínica

Trabajo recibido: marzo 2007

Trabajo aceptado: abril 2007 